\title{
V 700 Cygni and AW Virginis: Two W-type W UMa systems with spotted components ${ }^{\star}$
}

\author{
P.G. Niarchos ${ }^{1}$, M. Hoffmann ${ }^{2}$, and H.W. Duerbeck ${ }^{3,4}$ \\ 1 Section of Astrophysics, Department of Physics, University of Athens, Panepistimiopolis, GR-15784 Zografos, Athens, Greece \\ 2 Alter Weg 7, D-54570 Weidenbach, Germany \\ 3 European Southern Observatory, Casilla 19001, Santiago 19, Chile \\ 4 Astronomisches Institut, Wilhelm-Klemm-Str. 10, D-48149 Münster, Germany
}

Received April 10; accepted November 18, 1996

\begin{abstract}
A photometric analysis of the W UMa systems V 700 Cyg and AW Vir, based on photoelectric $B$ and $V$ light curves, is presented. Since no spectroscopic mass-ratio is available, the $q$-search method was applied to find the preliminary range of the mass-ratio in order to search for the final solution. First, an unspotted solution was carried out by using the unperturbed parts of the light curves and applying the DC program of the WD code. The final spotted solution was made by adopting spot models for the two systems. In the case of V $700 \mathrm{Cyg}$, two cool spots were placed on the primary (more massive, larger and cooler) component to explain the O'Connell effect (Max II fainter than MaxI) and a small deficit of light just before MaxI. In the case of AW Vir, one cool spot was placed on the primary component to explain the relatively large O'Connell effect. Both objects are W-type systems with partial eclipses; V $700 \mathrm{Cyg}$ is fairly evolved while AW Vir is rather unevolved.
\end{abstract}

Key words: stars: V 700 Cyg, AW Vir — binaries: eclipsing - starspots

\section{Introduction}

The variability of V 700 Cyg (Oklahoma Variable 26) was discovered by Whitney (1952), who classified it as a W UMa system with a period of 0.340048 days. Besides the minima given by Whitney, photographic ones were published by Busch et al. (1964) and Romano (1969), photoelectric ones by Hoffmann (1983) and by members of the

Send offprint requests to: P.G. Niarchos

* Tables 1, 2, 3, 4 only available: in electronic form at CDS via ftp to cdsarc.u-strasbg.fr $(130.79 .128 .5)$ or via http://cdsweb.u-strasbg.fr/Abstract.html
BAV (Agerer \& Hübscher 1995). Visual minima were observed by members of the BBSAG (Bulletins 23, 25, 39, $49,50,51$ and 56).

Eggen (1967) noted the presence of a close physical companion and derived the following magnitudes and colours:

$\begin{array}{lllll} & V_{\mathrm{E}} & B-V & U-B & E(B-V) \\ \text { V 700 Cyg } & 11.2 & +0.74 & +0.14 & +0.03 \\ \text { Companion } & 12.59+0.88 & +0.45 .\end{array}$

Its spectral type was determined to be $\mathrm{F} 2$ from objective prism spectra taken with the 10-inch Cooke refractor of the Leander McCormick Observatory (MacDonald 1964). Götz \& Wenzel (1967) derive a spectral type of A from Sonneberg Schmidt plates. Eggen's colours, however, indicate a much later spectral type. Since a good temperature estimate is essential for the analysis, Wenxian $\mathrm{Lu}$ (U. of Toronto) kindly took a CCD spectrum of V 700 Cyg, covering the range $380-450 \mathrm{~nm}$, with the Cassegrain spectrograph of the David Dunlap Observatory $1.9 \mathrm{~m}$ telescope. It was compared with main sequence star spectra from the atlas of Yamashita et al. (1977). The spectral type was found to be G5, in good agreement with the $U B V$ colours. The cause of the discrepancy of the old classifications is not clear; maybe the presence of the nearby visual companion has influenced the appearance of the spectrum of V 700 Cyg on the objective prism plates.

The variability of AW Vir was discovered by Hoffmeister (1935) on Babelsberg and Sonneberg plates. He found short-time light variability and suspected it to be an eclipsing binary. This was confirmed by Jensch (1936) who gave a list of minimum times, based on photographic and visual observations, and derived an ephemeris. Further minima were reported by Whitney (1955), Koch (1961), Hoffmann (1983), Lapasset \& Gómez (1995), and by members of the BBSAG (Bulletins 48, 67, 83, 88, 89, 


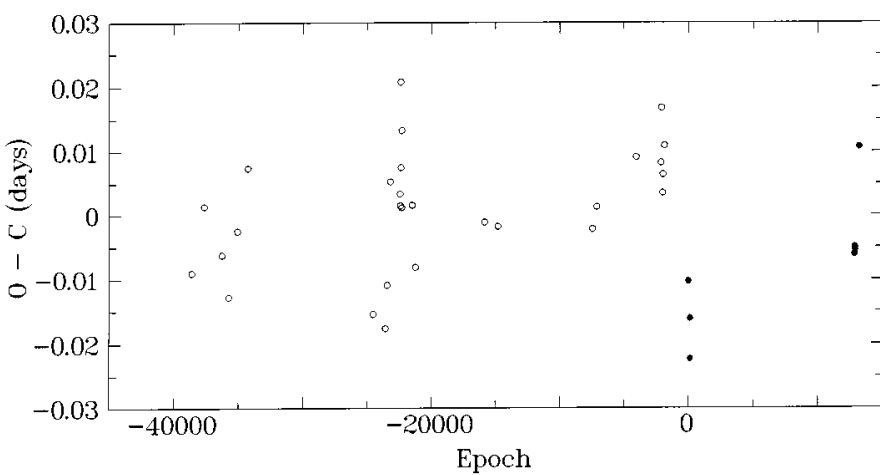

Fig. 1. $(\mathrm{O}-\mathrm{C})$ diagram of V $700 \mathrm{Cyg}$ based on the linear ephemeris. Photoelectric and CCD minima, as well as mean values of several minimum determinations are shown as filled circles, single visual and photographic minima are shown as empty circles

92, 95, 97, 98, 101, 104, 109; visual and CCD). Its spectral type was determined on objective prism spectra as F8 (MacDonald 1964). A synthetic light curve analysis of AW Vir was recently presented by Lapasset et al. (1996), who analysed $B$ and $V$ light curves collected in 1989 .

\section{Observations}

$B$ and $V$ photoelectric observations of both systems were carried out with a double channel photometer, attached to the $1.06 \mathrm{~m}$ Cassegrain reflector of Hoher List Observatory. V 700 Cyg was observed on 1982 July 12/13, August $25 / 26,27 / 28$ and September 8/9. The star BD $+38^{\circ} 4123$ was used as comparison star. AW Vir was observed on 1982 February 1/2, 20/21 and $21 / 22$. BD $+3^{\circ} 2773$ served as comparison star. The single observations were published by Hoffmann (1984).

\section{Times of minima. Period study}

A linear least squares solution of the available minima of V 700 Cyg, listed in Table 1 (available from cdsarc.ustrasbg.fr), yields the following solution:

$$
\begin{gathered}
\text { J.D.hel. }(\operatorname{Min} \mathrm{I})=2445163.4896+0.340045602 \cdot E . \\
\pm 0.0018 \pm 0.000000009 .
\end{gathered}
$$

A linear least squares solution of the available minima of AW Vir, listed in Table 2 (available from cdsarc.ustrasbg.fr), yields the following solution:

$$
\begin{gathered}
\text { J.D.hel. }(\text { Min I })=2445022.6496+0.35399707 \cdot E . \\
\pm 0.0015 \pm 0.00000005 .
\end{gathered}
$$

The $\mathrm{O}-\mathrm{C}$ diagram of V 700 Cyg (Fig. 1) indicates short-term period instability, while the diagram of AW Vir (Fig. 2) indicates only marginal period changes - something quite unusual for a W UMa system that has been under observation for so long.

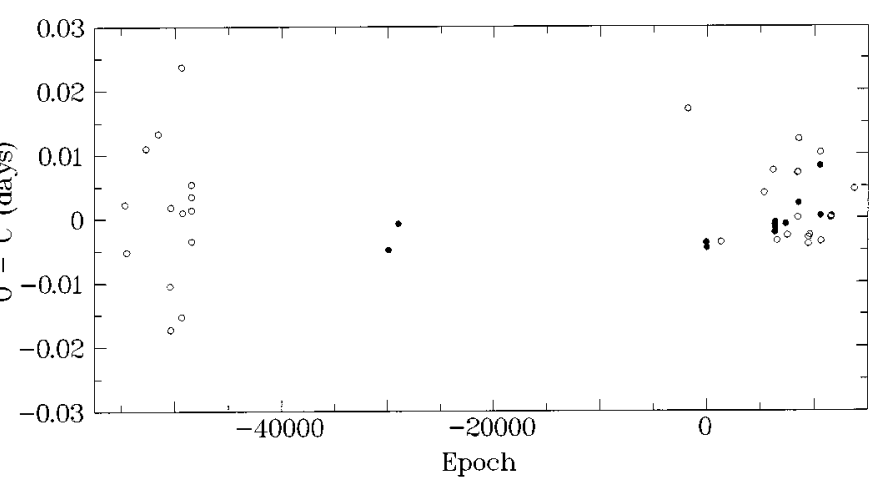

Fig. 2. $(\mathrm{O}-\mathrm{C})$ diagram of $\mathrm{AW}$ Vir based on the linear ephemeris. Photoelectric and CCD minima, as well as mean values of several minimum determinations are shown as filled circles, single visual and photographic minima are shown as empty circles

\section{Photometric solution}

The light curve analysis of both systems is quite difficult for the following reasons: (a) no spectroscopic mass-ratio is known, (b) the light curves show significant anomalies with O'Connell effect, and (c) the systems exhibit partial eclipses.

The light curves of V 700 Cyg reveal brightness fluctuations mainly around the maxima. The O'Connell effect is observed in both light curves and the magnitude difference between the two maxima is Max II - Max I = 0. 025 on the average for both light curves. A small deficiency of light is noted in the phase interval $0.13-0.24$, mainly in the $B$ band. The difference between the two minima is 0.230 in $B$ and 0.200 in $V$.

The light curves of AW Vir show brightness disturbances not only around the maxima, but also at other phases. A decrease in brightness is present in both $B$ and $V$ light curves in the phase interval $0.60-0.78$ and a small excess of light is seen in both curves in the phase interval $0.39-0.46$. Other minor light variations are present in other phase intervals. A small O'Connell effect is observed in both light curves. The magnitude difference between the two maxima is Max II - Max I $=0{ }^{\mathrm{m}} 025$ on the average for both light curves. The difference between the two minima is about $0 .{ }^{\mathrm{m}} 110$ in $B$ and $0 .{ }^{\mathrm{m}} 130$ in $V$.

With such anomalies in the light curves, the analysis of both systems is quite difficult. We tried to model the light curves by invoking spots on one or both components. The need to place cool (sunspot-like) and/or hot spots on the components of contact binaries to explain the light curve anomalies and the O'Connell effect, has been suggested by several investigators (e.g. Binnendijk 1960; Hilditch 1981; Linnell 1982; Van Hamme \& Wilson 1985; Milone et al. 1987; van't Veer \& Maceroni 1988, 1989; Maceroni et al. 1990). 


\subsection{Unspotted solution}

The light curve analysis was carried out by using the most recent (1993) version of the Wilson-Devinney (Wilson 1990) synthetic light curve code, which has the capacity of automatically adjusting the spots. In order to reduce computational time and to smooth the scatter around the maxima, normal points were formed from the individual observations and assigned weights equal to the number of observations per normal. The normal points of $\mathrm{V} 700$ Cyg are given in Table 3 and those of AW Vir in Table 4 (both tables are available from cdsarc.u-strasbg.fr). The mean standard deviation for normal points in $B$ and $V$ are $0{ }^{\mathrm{m}} 0143$ and $0{ }^{\mathrm{m}} 0142$ for $\mathrm{V} 700 \mathrm{Cyg}$, and 0. 0102 and 0. 0104 for AW Vir, respectively. Care has been taken to ensure a faithful resemblance of the normal points to the actual shape of the minima.

We proceeded to the unspotted solution by assuming that there are no spots on the components of the systems. Therefore the unperturbed parts of the light curves were used. In accordance with the light curve anomalies mentioned before, the observations in the phase intervals $0.13-0.24$ and $0.61-0.82$ were not included in the case of V 700 Cyg, while those in the interval $0.60-0.78$ were omitted in the case of AW Vir.

In the following, the subscripts $h$ and $\mathrm{c}$ refer to the hotter and cooler component, respectively. The shape of the light curve minima shows partial eclipses, so that we do not know a priori whether the systems belong to A or $\mathrm{W}$ type. In both cases the deeper primary minimum indicates that the hotter star is eclipsed at primary minimum. Therefore, the subscripts 1 and 2 in the DC program (with phase of conjunction $\phi_{0}=0.0$ ) are identical with $h$ and $c$, respectively. A preliminary set of input parameters for the DC program was obtained by the Binary Maker 2.0 program (Bradstreet 1993). The DC program was used in mode 3 . In the subsequent analysis the following assumptions were made:

Case V 700 Cyg: A mean surface temperature $T_{\mathrm{h}}=$ $5770 \mathrm{~K}$ according to the spectral type G5V; bolometric albedos $A_{\mathrm{h}}=A_{\mathrm{c}}=0.5$ and gravity darkening coefficients $g_{\mathrm{h}}=g_{\mathrm{c}}=0.32$ were assigned values typical for stars with convective envelopes; limb darkening coefficients $x_{\mathrm{h}}=x_{\mathrm{c}}=0.78$ in $B$ and $x_{\mathrm{h}}=x_{\mathrm{c}}=0.64$ in $V$ were taken from Al-Naimiy's (1978) tables; bolometric linear limb darkening coefficients $x_{\mathrm{h}}=x_{\mathrm{c}}=0.505$ were taken from Van Hamme (1993); third light was assumed to be $\ell_{3}=0.0$.

Case $A W$ Vir: A mean surface temperature $T_{\mathrm{h}}=$ $6200 \mathrm{~K}$ according to the spectral type F8V; bolometric albedos and gravity darkening coefficients were assigned the same values as for V $700 \mathrm{Cyg}$; limb darkening coefficients $x_{\mathrm{h}}=x_{\mathrm{c}}=0.75$ in $B$ and $x_{\mathrm{h}}=x_{\mathrm{c}}=0.61$ in $V$ were taken from Al-Naimiy's tables; bolometric linear limb darkening coefficients $x_{\mathrm{h}}=x_{\mathrm{c}}=0.49$ were taken again from Van Hamme (1993); third light was assumed to be $\ell_{3}=0.0$.

The adjustable parameters were in both cases: the phase of conjunction $\phi_{0}$, the inclination $i$, the temperature $T_{\mathrm{c}}$, the non-dimensional potential $\Omega_{\mathrm{h}}\left(=\Omega_{\mathrm{c}}\right)$, the monochromatic luminosity $L_{\mathrm{h}}$ and the mass-ratio $q=$ $m_{\mathrm{c}} / m_{\mathrm{h}}$. The quantity $\phi_{0}$ was adjusted only in the first few iterations, since it showed no tendency to vary significantly. The lack of a spectroscopic mass-ratio for both systems led us to search for the solution with several fixed values for the mass-ratio $q$ in the range $0.2-4$. The values of $q<1$ correspond to a transit at the primary (A-type system) and those of $q>1$ to an occultation (W-type system). The lowest values of the sum $\Sigma(\text { res })^{2}$ of the weighted squares of the residuals occured at $q=0.8$ and $q=1.6$ for the case of $\mathrm{V} 700 \mathrm{Cyg}$ and at $q=0.8$ and $q=1.2$ for AW Vir, with almost equal values in both cases for each system. Figures 3 and 4 show the fit parameter $\Sigma(\text { res })^{2}$ as a function of the mass-ratio $q$. The range in $q$ for $\mathrm{V} 700$ Cyg is $0.4-2.8$, since for values of $q$ outside this range no convergent acceptable solution could be obtained.

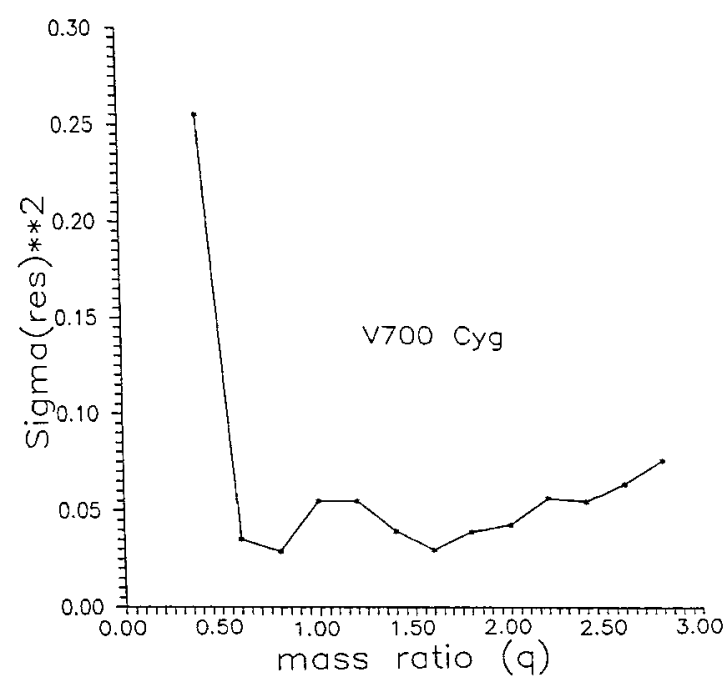

Fig. 3. V 700 Cyg: The fit parameter $\Sigma(\text { res })^{2}$ as a function of the mass-ratio $q$

In order to find the final unspotted solution we continued the analysis by applying the DC program for the above values of $q$, treating $q$ as a free parameter. The solutions for $\mathrm{V} 700 \mathrm{Cyg}$ converged to $q=0.8895$ and $q=1.5699$ with values of $\Sigma(\text { res })^{2} 0.0330$ and 0.0319 , respectively. In the case of AW Vir the solutions converged to $q=0.8006$ and $q=1.2222$ and the corresponding values of $\Sigma(\text { res })^{2}$ were found to be 0.0210 and 0.0202 . Of these two solutions ( $\mathrm{A}$ and $\mathrm{W}$-type for each system), we finally adopted the W-type solutions by taking into account (a) the better fit of the W-type solution and (b) the large mass-ratio of the A-type solution, which would be unusual for an 
Table 5. Light curve solutions of V 700 Cyg and AW Vir

\begin{tabular}{|c|c|c|c|c|c|}
\hline Parameter & $\begin{array}{l}\text { V } 700 \mathrm{Cyg} \\
\text { unspotted } \\
\text { solution }\end{array}$ & $\begin{array}{l}\text { V } 700 \text { Cyg } \\
\text { spotted } \\
\text { solution }\end{array}$ & $\begin{array}{c}\text { AW Vir } \\
\text { unspotted } \\
\text { solution }\end{array}$ & $\begin{array}{l}\text { AW Vir } \\
\text { spotted } \\
\text { solution }\end{array}$ & $\begin{array}{c}\text { AW Vir } \\
\text { unspotted solution } \\
\text { (Lapasset et al. 1996) }\end{array}$ \\
\hline$\overline{\phi_{0}}$ & $0.9998 \pm 0.0003$ & $0.9998 \pm 0.0003$ & $0.0018 \pm 0.0003$ & $0.0010 \pm 0.0002$ & \\
\hline$i$ (degrees) & $80.268 \pm 0.183$ & $79.856 \pm 0.173$ & $81.357 \pm 0.195$ & $81.193 \pm 0.167$ & $82.98 \pm 0.09$ \\
\hline$g_{\mathrm{h}}\left(=g_{\mathrm{c}}\right)$ & $0.32^{*}$ & $0.32^{*}$ & $0.32^{*}$ & $0.32^{*}$ & $0.32^{*}$ \\
\hline$T_{\mathrm{h}}(\mathrm{K})$ & $5770^{*}$ & $5770^{*}$ & $6200^{*}$ & $6200^{*}$ & $6000^{*}$ \\
\hline$T_{\mathrm{c}}(\mathrm{K})$ & $5351 \pm 10$ & $5396 \pm 9$ & $5945 \pm 12$ & $5872 \pm 10$ & $5980 \pm 5$ \\
\hline$A_{\mathrm{h}}\left(=A_{\mathrm{c}}\right)$ & $0.5^{*}$ & $0.5^{*}$ & $0.5^{*}$ & $0.5^{*}$ & $0.5^{*}$ \\
\hline$\Omega_{\mathrm{h}}\left(=\Omega_{\mathrm{c}}\right)$ & $4.4798 \pm 0.0164$ & $4.4185 \pm 0.0218$ & $4.0500 \pm 0.0172$ & $4.1991 \pm 0.0081$ & $3.207 \pm 0.003$ \\
\hline$q=m_{\mathrm{c}} / m_{\mathrm{h}}$ & $1.5699 \pm 0.0097$ & $1.5346 \pm 0.0105$ & $1.2222 \pm 0.0042$ & $1.3150 \pm 0.0058$ & 1.48 \\
\hline$L_{\mathrm{h}} /\left(L_{\mathrm{h}}+L_{\mathrm{c}}\right)(B)$ & $0.5115 \pm 0.0042$ & $0.5040 \pm 0.0034$ & $0.5111 \pm 0.0040$ & $0.5115 \pm 0.0030$ & 0.585 \\
\hline$L_{\mathrm{h}} /\left(L_{\mathrm{h}}+L_{\mathrm{c}}\right)(V)$ & $0.4899 \pm 0.0035$ & $0.4848 \pm 0.0028$ & $0.5002 \pm 0.0035$ & $0.4972 \pm 0.0025$ & 0.584 \\
\hline$x_{\mathrm{h}}\left(=x_{\mathrm{c}}\right)(B)$ & $0.78^{*}$ & $0.78^{*}$ & $0.75^{*}$ & $0.75^{*}$ & $0.709^{*}$ \\
\hline$x_{\mathrm{h}}\left(=x_{\mathrm{c}}\right)(V)$ & $0.64^{*}$ & $0.64^{*}$ & $0.61^{*}$ & $0.61^{*}$ & $0.573^{*}$ \\
\hline$x_{\mathrm{h}}\left(=x_{\mathrm{c}}\right)$ (bolo) & $0.505^{*}$ & $0.505^{*}$ & $0.49^{*}$ & $0.49^{*}$ & \\
\hline$\%$ overcontact & $26 \%$ & $27 \%$ & $9 \%$ & $8 \%$ & $2.1 \%$ \\
\hline$r_{\mathrm{h}}($ pole $)$ & $0.3344 \pm 0.0012$ & $0.3373 \pm 0.0015$ & $0.3454 \pm 0.0010$ & $0.3386 \pm 0.0007$ & 0.332 \\
\hline$r_{\mathrm{h}}$ (side) & $0.3522 \pm 0.0014$ & $0.3556 \pm 0.0018$ & $0.3630 \pm 0.0012$ & $0.3554 \pm 0.0008$ & 0.348 \\
\hline$r_{\mathrm{h}}($ back $)$ & $0.3945 \pm 0.0023$ & $0.3987 \pm 0.0026$ & $0.3978 \pm 0.0016$ & $0.3899 \pm 0.0013$ & 0.383 \\
\hline$r_{\mathrm{c}}($ pole $)$ & $0.4089 \pm 0.0022$ & $0.4081 \pm 0.0029$ & $0.3789 \pm 0.0028$ & $0.3842 \pm 0.0012$ & 0.391 \\
\hline$r_{\mathrm{c}}($ side $)$ & $0.4356 \pm 0.0029$ & $0.4348 \pm 0.0038$ & $0.4001 \pm 0.0036$ & $0.4059 \pm 0.0016$ & 0.414 \\
\hline$r_{\mathrm{c}}($ back $)$ & $0.4718 \pm 0.0043$ & $0.4717 \pm 0.0058$ & $0.4331 \pm 0.0055$ & $0.4382 \pm 0.0023$ & 0.446 \\
\hline$\Sigma_{(\mathrm{res})^{2}}$ & 0.0319 & 0.0338 & 0.0202 & 0.0157 & \\
\hline$\overline{\mathcal{M}_{\mathrm{h}} / \mathcal{M}_{\odot}}$ & & 0.60 & & 0.84 & \\
\hline $\mathcal{M}_{\mathrm{c}} / \mathcal{M}_{\odot}$ & & 0.92 & & 1.11 & \\
\hline$R_{\mathrm{h}} / R_{\odot}$ & & 0.86 & & 0.95 & \\
\hline$R_{\mathrm{c}} / R_{\odot}$ & & 1.04 & & 1.08 & \\
\hline $\log \left(L_{\mathrm{h}} / L_{\odot}\right)$ & & -0.13 & & 0.08 & \\
\hline $\log \left(L_{\mathrm{c}} / L_{\odot}\right)$ & & -0.08 & & 0.10 & \\
\hline
\end{tabular}

always performed after obtaining a convergent solution to ensure its stability. The final results of the spotted solution of V 700 Cyg and AW Vir are given in Table 5, and the corresponding theoretical light curves are shown as solid lines in Figs. 5 and 6 , respectively. The final spot parameters for the two systems are given in Table 6 . The final spotted solution for V $700 \mathrm{Cyg}$ (where the spots were adjusted) places the two spots on the primary in positions where both are visible at phase 0.0 and hence should introduce perturbations in that phase region. These perturbations are quite small, of the order of the statistical error lines (see Sect. 5 for a discussion), because the temperature factor of the spots are very close to unity. The $\mathrm{O}-\mathrm{C}$ differences between the observed and calculated points for the unspotted and spotted solutions for the two systems are shown in Figs. 7 and 8, respectively.

\section{Discussion and conclusions}

Although the available information about the systems V 700 Cyg and AW Vir is based only on photometry and spectral classification, the adopted spot models fit satisfactorily the observed light curves. The two minima have
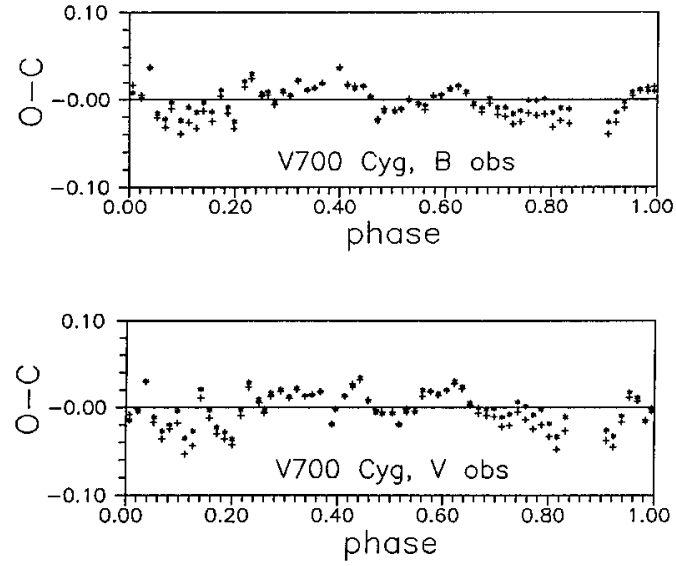

Fig. 7. The light curve $(\mathrm{O}-\mathrm{C})$ residuals for $\mathrm{V} 700 \mathrm{Cyg}$ in $B$ and $V$ band. Crosses refer to unspotted solution; asterisks refer to spotted solution

unequal depths, and a temperature difference of a few hundred degrees between the components is expected. The final solution yields a temperature difference $\Delta T \approx 374 \mathrm{~K}$ 

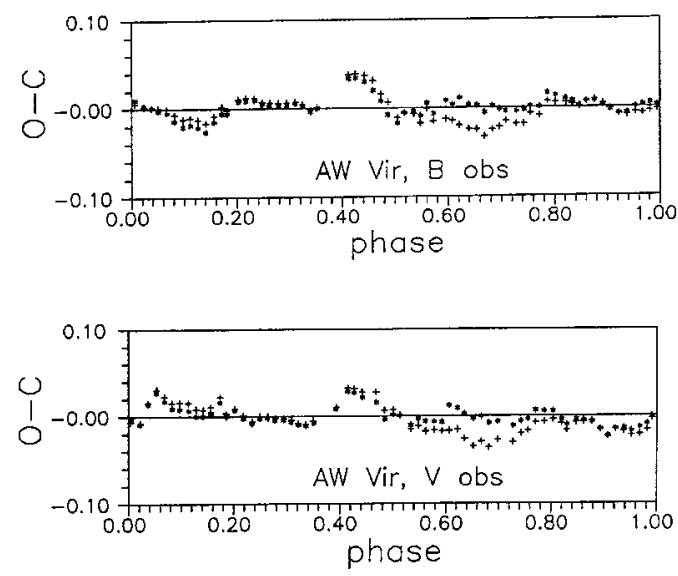

Fig. 8. The light curve $(\mathrm{O}-\mathrm{C})$ residuals for $\mathrm{AW}$ Vir in $B$ and $V$ band. Crosses refer to unspotted solution; asterisks refer to spotted solution

Table 6. Spot parameters

\begin{tabular}{l|cc|c}
\hline & $\begin{array}{c}\text { V 700 Cyg } \\
\text { spot } 1\end{array}$ & $\begin{array}{c}\text { V 700 Cyg } \\
\text { spot 2 }\end{array}$ & $\begin{array}{c}\text { AW Vir } \\
\text { spot } 1\end{array}$ \\
\hline$b$ (degrees) & 90 & 90 & 80 \\
$l$ (degrees) & $241.77 \pm 6.41$ & $123.20 \pm 4.58$ & $309.51 \pm 4.98$ \\
$R$ (degrees) & $20.16 \pm 0.84$ & $19.60 \pm 1.07$ & $14.56 \pm 0.32$ \\
T.F. & $0.945 \pm 0.005$ & $0.952 \pm 0.006$ & $0.720 \pm 0.028$ \\
\hline
\end{tabular}

between the components of $\mathrm{V} 700 \mathrm{Cyg}$, and $\Delta T \approx 320 \mathrm{~K}$ between those of AW Vir. According to our W-type solution, the larger and more massive component of both systems is also the cooler one, while the smaller and less massive is the hotter component. The thermal-decoupling degree, defined as $D T=\left(T_{1}-T_{2}\right) / T_{1}$ (Lipari \& Sistero 1988), where $T_{1}$ corresponds to the temperature of the larger and more massive component and $T_{2}$ to that of the smaller and less massive one, yields values -0.07 and -0.05 for V 700 Cyg and AW Vir, respectively. These values place the two systems among the $\mathrm{W}$-type systems in Figs. 2 and 3 of Lipari \& Sistero (1988). In the previous expression for $D T$ we have replaced, according to our $\mathrm{W}$ type solution, $T_{1}=T_{\mathrm{c}}$ and $T_{2}=T_{\mathrm{h}}$.

We succeeded to model the light curves of V $700 \mathrm{Cyg}$ by invoking two cool spots on the larger (cooler, more massive) component, while for AW Vir one cool spot on the larger (cooler, more massive) component, was sufficient to obtain a satisfactory fit to the observations. As far as the uniqueness of our model is concerned, it should be pointed out that solutions with different spot combinations may equally well fit the observations. According to Maceroni et al. (1990) and Maceroni \& van't Veer (1993) this is a general problem of light curve fitting with spot models. On the basis of this argument, we tried to use the simplest model with a physical meaning.
Given that a unique solution can hardly be obtained for partially eclipsing systems with unknown spectroscopic mass-ratio, we can invoke an independent check of the quality of the derived (unspotted and spotted) solutions by considering the residuals for each light curve. Barone et al. (1993) pointed out that, according to the Chauvenet hypothesis, at least $68 \%$ of the points should be in the band defined by the statistical error lines $\left(-S_{\text {er }}, S_{\text {er }}\right)$, where $S_{\text {er }}$ is given by Eq. (7) of Barone et al. (1993). In the case of V $700 \mathrm{Cyg}$ we find $S_{\text {er }}=0.0177$ for $B$ and $S_{\text {er }}=0.0178$ for $V$. For the unspotted solution (with phase intervals $0.13-0.24$ and $0.61-0.82$ excluded) $68 \%$ of the $\mathrm{O}-\mathrm{C}$ points in $B$ and $67 \%$ in $V$ lie inside the statistical error lines. When the whole light curve is considered, the Chauvenet hypothesis is not fulfilled. In the spotted solution (where all the points are included) $82 \%$ of the $\mathrm{O}-\mathrm{C}$ points in $B$ and $66 \%$ of the points in $V$ are within the error lines. Therefore, both the unspotted and spotted solutions fulfill the Chauvenet hypothesis marginally making thus both solutions acceptable.

In the case of AW Vir, the error lines are $S_{\text {er }}=0.0139$ in $B$ and $S_{\text {er }}=0.0143$ in $V$. Similarly, for the unspotted solution (with phase interval $0.60-0.78$ excluded) $85 \%$ of the $\mathrm{O}-\mathrm{C}$ points in $B$ and $68 \%$ in $V$ lie inside the error lines: if we consider the whole light curve, the corresponding numbers are $72 \%$ and $51 \%$ in $B$ and $V$, respectively. For the spotted solution $83 \%$ of the $\mathrm{O}-\mathrm{C}$ points in $B$ and $84 \%$ in $V$ are within the statistical error lines. These data indicate that both solutions, the unspotted and spotted one, are acceptable, since both fulfill the Chauvenet hypothesis.

After our study had been submitted, a photometric analysis of AW Vir was published by Lapasset et al. (1996). They used the WD code to analyse their $B, V$ light curves, which do not show noticeable asymmetries or irregularities. An unspotted solution was found on the basis of the best fit, which formally corresponds to an Atype configuration, although the values of some physical parameters - mass-ratio, degree of contact and spectral type - are typical for W-type systems. The authors conclude they are unable to assess unambiguously the subtype of this system by photometric evidence only. Since their identification of minimum I and II is the same as in the present paper, we are confident to say that AW Vir oscillates between states of $D T$ between 0 and a negative value.

Our solution is based on $B, V$ light curves, obtained 7 years before the observations of Lapasset et al. They clearly show a brightness deficiency in the phase interval $0.60-0.78$, and require the application of a spot model. Our W-type solution is based not only on the best fit, but also on the similarity of some basic physical parameters of AW Vir to those typical for W-type systems. Despite the different subtypes found in the two analyses, the geometrical elements (inclination and relative radii) are very similar in the two solutions (see Table 5). Lapasset et al. 
assumed a spectral type G0 for the primary component on the basis of $U B V$ colours; since the photometric data given in their Table 2 are apparently misprinted, it is difficult to judge whether this spectral type is to be preferred over MacDonald's (1964) spectral classification.

Three-dimensional pictures of the spotted models of V 700 Cyg and AW Vir are shown in Figs. 9 and 10. Cross-sectional surface outlines of the two systems with their inner and outer critical Roche surfaces are shown in Figs. 11 and 12.

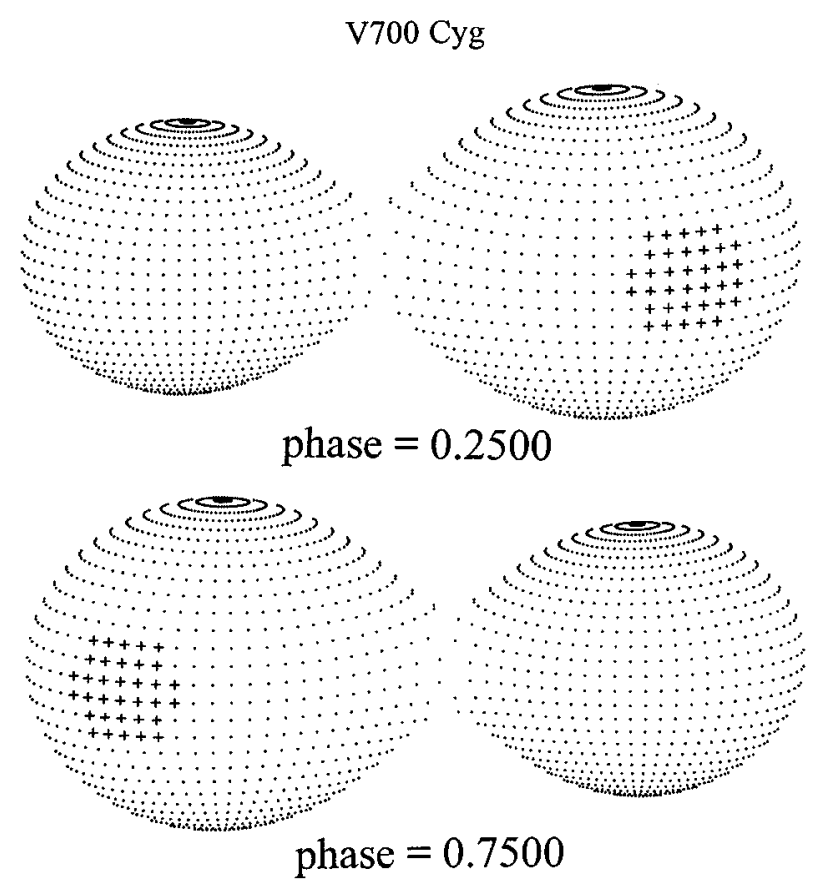

Fig. 9. A three-dimensional model of V 700 Cyg for phases 0.25 (upper plot) and 0.75 (lower plot)

The present results are based on photometry only, since no radial velocities of the systems exist so far. Nevertheless it is interesting to speculate on the evolution status by using the absolute parameters of the systems. These were computed by the LC program of the WD code and standard relations, where the parameters found in the spotted solutions were used as input parameters, and are listed in Table 5.

The absolute elements were used to estimate the evolutionary status of the two systems by means of the massradius (MR), mass-luminosity (ML) and HR diagrams of Hilditch et al. (1988), who showed that the mass-radius diagram is the principal indicator of the evolutionary state. The primary components of V 700 Cyg and AW Vir lie in the region between ZAMS and TAMS, which is occupied by the primaries of other W-type W UMa systems. The position of V $700 \mathrm{Cyg}$, quite close to the TAMS, indicates that this system is somewhat evolved while AW Vir
AW Vir
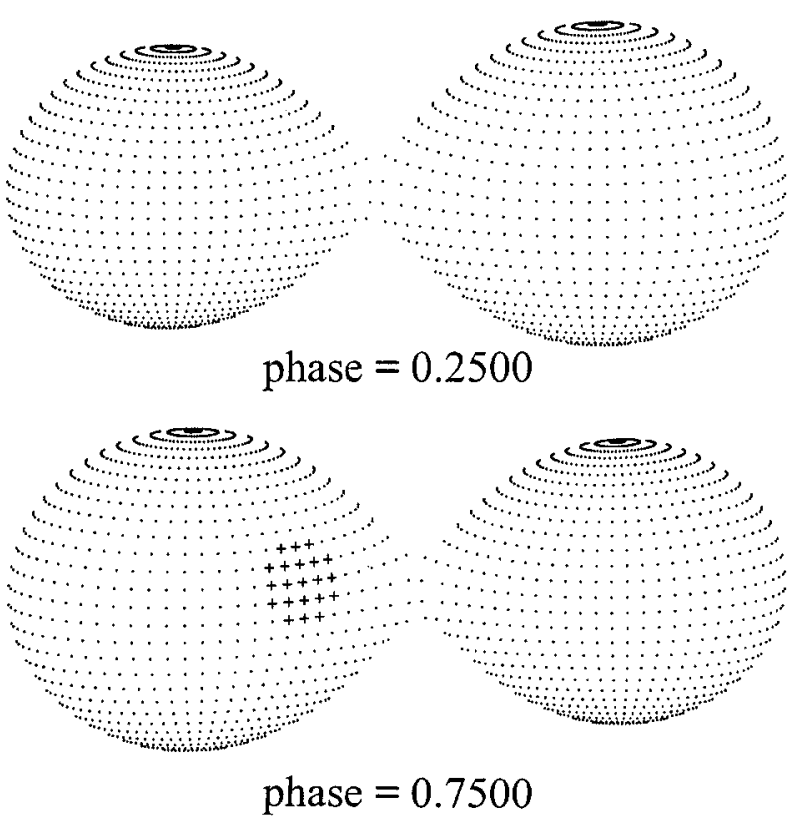

Fig. 10. A three-dimensional model of AW Vir for phases 0.25 (upper plot) and 0.75 (lower plot)

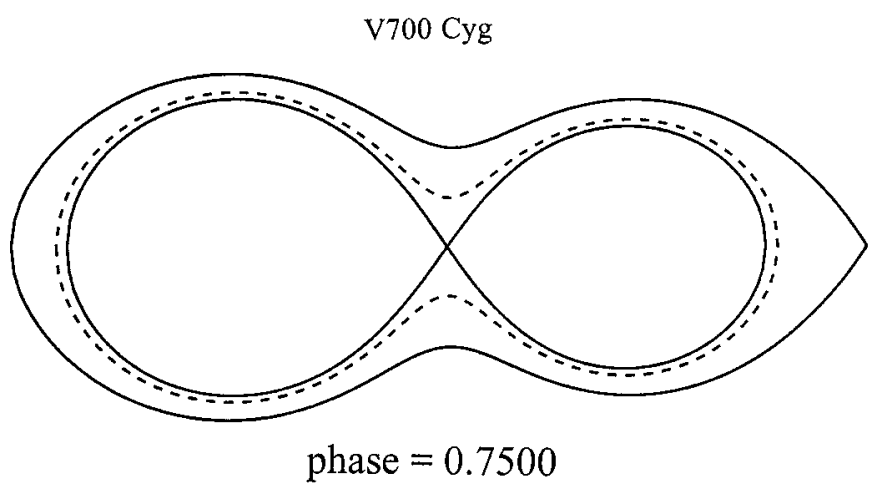

Fig. 11. Cross-sectional surface outline of V 700 Cyg (dashed line). The solid lines are the Roche critical surfaces.

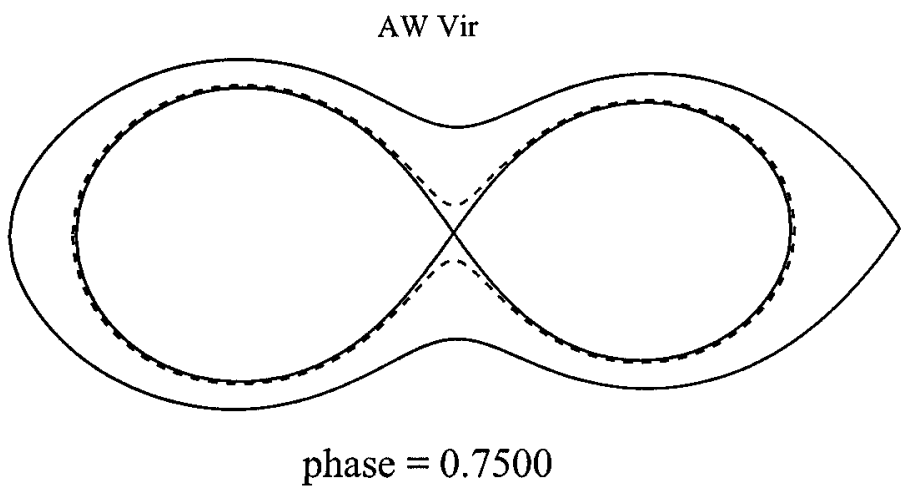

Fig. 12. Cross-sectional surface outline of AW Vir (dashed line). The solid lines are the Roche critical surfaces 
is rather unevolved. In both cases, the secondary component lies in the region where other secondaries of W-type W UMa systems are found, and has a larger radius than expected for the ZAMS mass (Hilditch et al. 1988).

The derived W-type solutions, supported by the thermal-decoupling degree of the systems, successfully reproduce the observations and are consistent with the MR, ML and HR diagrams of Hilditch et al. (1988). But more definite conclusions about the evolutionary status of V 700 Cyg and AW Vir can only be drawn by means of additional photometric and spectroscopic observations of the systems.

Acknowledgements. We are especially grateful to Wenxian $\mathrm{Lu}$ for taking a spectrum of $\mathrm{V} 700 \mathrm{Cyg}$ on short notice. We thank W. Van Hamme for useful discussions, the referee, F. van't Veer, for his comments on the manuscript, and H. Busch for communicating data from the Hartha Circular. The 3-D pictures and cross-sectional surface outlines were made by D.H. Bradstreet's Binary Maker 2.0. H.W.D. was supported by a ESO Senior Visitor fellowship.

\section{References}

Agerer F., Hübscher J., 1995, IBVS 4222

Al-Naimiy H.M., 1978, ApSS 56, 219

Barone F., Di Fiore L., Milano L., Russo G., 1993, ApJ 407, 237

Bradstreet D.H., 1993, Binary Maker 2.0 User Manual

Binnendijk L., 1960, AJ 65, 358

Busch H., et al., 1964, Hartha Beob.-Zirk. 27-28

Eggen O.J., 1967, Mem RAS 70, 111
Götz W., Wenzel W., 1967, Mitt. Veränderl. Sterne Sonneberg 4,67

Hilditch R.W., 1981, MNRAS 196, 305

Hilditch R.W., King D.J., McFarlane T.M., 1988, MNRAS 231, 341

Hoffmann M., 1983, IBVS 2344

Hoffmann M., 1984, Veröff. Astr. Inst. Univ. Bonn No. 96

Hoffmeister C., 1935, Astr. Nachr. 255, 401

Jentsch A., 1936, Astr. Nachr. 256, 279

Koch R.H., 1961, AJ 66, 35

Lapasset E., Gómez M., 1995, IBVS 4144

Lapasset E., Gómez M., Farinãs R., 1996, PASP 108, 332

Linnell A.P., 1982, ApJS 50, 85

Lipari S.L., Sistero R.F., 1988, PASP 100, 377

MacDonald D.D., 1964, Publ. Leander McCormick Obs. 12, pt V, p. 51

Maceroni C., Van Hamme W., van't Veer F., 1990, A\&A 234, 177

Maceroni C., van't Veer F., 1993, A\&A 277, 515

Milone E.F., Wilson R.E., Hrivnak B.J., 1987, ApJ 319, 325

Mullan D.G., 1975, ApJ 245, 650

Romano G., 1969, Mem. S. A. It. 40, 375

Van Hamme W., 1993, AJ 106, 2096

Van Hamme W., Wilson R.E., 1985, A\&A 152, 25

van't Veer F., Maceroni C., 1988, A\&A 199, 183

van't Veer F., Maceroni C., 1989, A\&A 220, 128

Wilson R.E., 1990, ApJ 356, 613

Wilson R.E., Biermann P., 1976, A\&A 48, 349

Whitney B.S., 1952, AJ 56, 206

Whitney B.S., 1955, AJ 60, 453

Yamashita Y., Nariai K., Norimoto Y., 1977, An Atlas of Representative Stellar Spectra, Tokyo. University of Tokyo Press 